\title{
CONHECIMENTO DOS PROFISSIONAIS DE ENFERMAGEM EM SALA DE VACINA: ANÁLISE DA PRODUÇÃO CIENTÍFICA
}

\section{KNOWLEDGE OF PROFESSIONAL NURSES IN THE VACCINE ROOM: ANALYSIS OF SCIENTIFIC PRODUCTION}

\author{
Natália Pereira Marinelli ${ }^{1}$ \\ Khelyane Mesquita Carvalho \\ Telma Maria Evangelista Araújo ${ }^{3}$
}

Resumo: A enfermagem desempenha papel fundamental na sala de vacina necessitando de conhecimento adequado. O objetivo desta pesquisa foi identificar o conhecimento sobre as atividades cotidianas de sala de vacina, apontados na literatura. Realizou-se revisão integrativa, nas bases de dados LILACS, PubMed, CINAHL, Scopus, Cochrane e na biblioteca virtual SCIELO, a partir da questão: Quais os entraves apontados na literatura, sobre o conhecimento dos profissionais de enfermagem de sala de vacina? Dentre os artigos publicados, 15 associavam-se ao objetivo proposto. Observou-se que os estados do Piauí tem o maior número de publicações sobre a temática e a abordagem metodológica mais utilizada foi a quantitativa. $A$ análise da amostra mostrou repedidas vezes conhecimento inadequado dos profissionais em sala de vacina, a maioria relacionada à conservação dos imunobiológicos. Identificou-se demanda urgente na qualificação dos profissionais de enfermagem de sala de vacina. Percebe-se que todos os artigos analisados evidenciam descumprimento das ações referentes ao manual de vacinação, apontando necessidade de processo contínuo e sistemático de supervisão e educação permanente.

Palavras-chave: conhecimento; vacina; enfermagem; imunização.

Abstract: Nurses play a fundamental role in the vaccination room and need adequate knowledge. The objective of this research was to identify the knowledge about the daily activities of the vaccine room, reported in the literature. An integrative review was conducted in databases LILACS, PubMed, CINAHL, Scopus, Cochrane, and virtual library SCIELO for the question: What are the barriers identified in literature on knowledge of nursing professionals about the vaccine room? Among the published articles, 15 covered this objective. It was observed that the state of Piaui has the largest number of publications on the subject, and the most common methodological approach was quantitative. The analysis of the sample showed many times the inadequate knowledge of professionals in a vaccine room, mostly related to conservation of biopharmaceuticals. The urgent demand for the qualification of vaccine room nurses was identified. All the articles analyzed found noncompliances with the vaccination manual, which points to the need for continuous and systematic process of supervision and continuing education.

Keywords: knowledge; vaccine; nursing; immunization.

\footnotetext{
1 Doutoranda em Tecnologia e Mestre em Engenharia Biomédica - Universidade do Vale do Paraíba - Univap, Brasil. Docente da Universidade Federal do Piauí - UFPI, Brasil. E-mail: enfnatmarinelli@hotmail.com.

${ }^{2}$ Doutoranda e Mestre em Enfermagem - UFPI, Brasil. Docente da UFPI. E-mail: khelyanemc@gmail.com.

${ }^{3}$ Doutora em Enfermagem em Saúde Coletiva - Universidade Federal do Rio de Janeiro - UFRJ, Brasil. Professora Adjunta - UFPI. E-mail: telmaevangelista@gmail.com.
} 


\section{INTRODUÇÃO}

A imunização constitui uma das medidas mais eficazes na prevenção de doenças e requer conhecimento adequado que garanta sua qualidade efetiva para não comprometer $\mathrm{e}$ nem abalar a credibilidade da vacinação. É importante destacar que essa prática representa uma das principais ações de intervenção em saúde pública no controle de doenças provocadas por agentes imunizáveis, de modo que as vacinas dão provas incontestes de sua eficácia (BRASIL, 2013).

Todavia o sucesso da vacinação não pode levar em consideração apenas o cumprimento da cobertura vacinal, e sim das condições ideais de armazenamento, preparo e administração dessas vacinas, fazendo-se necessária a verificação do conhecimento dos profissionais, que desempenham suas funções na sala de vacina, que irá impactar na qualidade do serviço e do produto ofertado à população.

Estudos realizados no Brasil têm evidenciado a falta de conhecimento dos profissionais sobre intervalos de temperatura adequados para a conservação, inexistência de termômetros ou monitoramento diário de temperaturas, detecção de exposição frequente dos produtos a extremos de temperatura $\left(<0^{\circ} \mathrm{C}\right.$ e $\left.>10^{\circ} \mathrm{C}\right)$ durante 0 transporte e 0 armazenamento, organização inadequada dos refrigeradores e não exclusividade dos mesmos para estocar vacinas (FEITOSA; FEITOSA; CORIOLANO, 2010; QUEIROZ et al., 2009; OLIVEIRA et al., 2009).

Essa realidade configura um problema, visto que a prática de vacinação não deseja apenas atingir as metas das campanhas ou de vacinação de rotina, no que diz respeito à cobertura vacinal, e sim a soro conversão propriamente dita, que garantirá a proteção contra agravos imunopreveníveis.

O Programa Nacional de Imunização (PNI) é responsável por fornecer apoio técnico, supervisionar e avaliar a execução das atividades de vacinação em todo o território nacional, buscando manter a qualidade dos imunobiológicos, que podem sofrer alterações de seu poder imunogênico quando não garantida correta operacionalização do processo (BRASIL, 2011).

O papel da Enfermagem na sala de vacina é definir as ações que são de sua responsabilidade nesse contexto, contribuindo para o controle e/ou erradicação de agravos evitáveis por imunizantes com execução correta de toda a política no que diz respeito à conservação dos imunobiológicos, correta administração e preparo da vacina, conduta frente aos efeitos adversos, preenchimento correto dos impressos e educação continuada para profissionais.

Partindo desse pressuposto, faz-se necessário o levantamento de dados que evidencie $\mathrm{o}$ conhecimento adequado $\mathrm{e}$, consequentemente, prática adequada dos profissionais de sala de vacina, o que permitirá a proposta de algumas estratégias que possam minimizar as falhas para cumprimento efetivo das normas estabelecidas pelo PNI. 
Acompanhar o desenvolvimento de uma área do conhecimento por meio da análise de sua produção científica é um modo interessante de identificar os entraves de determinadas temáticas e apontar caminhos de crescimento e aprimoramento de outras. A análise da produção bibliográfica tem por objetivo avaliar e descrever estudos, levando-se em consideração o conteúdo das publicações, analisando também a qualidade do que vem sendo publicado (SOUZA; SILVA; CARVALHO, 2010).

Nesse contexto, a relevância deste estudo centra-se no objetivo de identificar as evidências científicas sobre o conhecimento da equipe de enfermagem em sala de vacina, apontados na literatura. É importante ressaltar que o presente estudo contribui para que se reflita sobre as condutas inadequadas em sala de vacina, fornecendo subsídios para ações educativas de aperfeiçoamento das práticas relacionadas à imunização, valorizando o cuidado e a orientação de enfermagem.

\section{METODOLOGIA}

Com a finalidade de alcançar o objetivo proposto, o método de revisão integrativa da literatura foi selecionado para a realização deste estudo. Adotou-se, para tanto, a sequência das seguintes etapas: 1) Seleção da questão norteadora, na temática da revisão; 2) Determinação dos critérios de inclusão e exclusão dos artigos e seleção dos estudos para composição da amostra; 3) Definição das informações a serem extraídas dos estudos selecionados; 4) Análise dos estudos que integram a amostra; 5) Interpretação dos resultados; e 6) Relato da revisão (SOUZA; SILVA; CARVALHO, 2010).

A elaboração dessa revisão integrativa partiu da seguinte questão norteadora: Quais os entraves apontados, na literatura, sobre o conhecimento dos profissionais de enfermagem de sala de vacina?

Os critérios de inclusão que nortearam a seleção da amostra foram: serem artigos científicos disponíveis na íntegra, eletronicamente, que abordassem questões pertinentes ao conhecimento dos profissionais de enfermagem que atuam em salas de vacina, e artigos redigidos nos idiomas inglês, português ou espanhol. É válido destacar que não houve restrições quanto ao período de publicação ou nível de evidência do artigo.

A busca dos estudos aconteceu em março de 2014, por meio do acesso online às seguintes bases de dados: LILACS (Literatura Latino-Americana e do Caribe em Ciências da Saúde), PubMed (Public/Publish Medline), CINHAL (Cumulative Index to Nursing and Allied Health Literature), SCIELO (Scientific Eletronic Library Online) Scopus e Cochrane. A pesquisa se deu a partir das palavras utilizadas como descritores no DeCS (Descritores em Ciências da Saúde): Conhecimento, vacina, enfermagem e Imunização, dos quais apenas 15 serviram como base para a construção do trabalho, em virtude da classificação e agrupamento dos itens.

Após busca, os artigos foram lidos e classificados de acordo com: ano de publicação, 
local realizado, delineamento metodológico, ênfase do estudo e conclusão.

A análise e discussão dos dados foram realizadas mediante avaliação crítica do material, tomando como base as informações nela contidas. Vale ressaltar que periódicos científicos disponíveis via internet dessas bases de dados são previamente selecionados em função de rigorosos critérios de admissão e permanência definidos de acordo com parâmetros internacionalmente aceitos, devendo, portanto, a veracidade do conteúdo ser de responsabilidade dos pesquisadores.

Para melhor visualização dos resultados e facilitar a discussão, foi realizada análise estatística e calculadas as frequências e os percentuais simples, sendo posteriormente agrupados em tabelas, quadros e gráficos.

\section{RESULTADOS}

Foram encontrados 15 artigos, relacionados com os descritores supracitados, dos quais, a grande maioria não aborda, de forma clara, todos os aspectos propostos neste estudo.

Em se tratando do ano de publicação podemos observar, no Gráfico 1, artigos publicados com até 10 anos, uma realidade que retrata um número muito pequeno de pesquisas sobre essa temática em uma década, principalmente se levarmos em consideração que não foram todos realizados pela enfermagem. Podemos destacar que a maioria das publicações refere-se ao período de 2009 a 2012.

\section{Gráfico 1 - Distribuição dos estudos revisados, segundo o período de publicação. Teresina/PI}

\section{Número de Publicações}

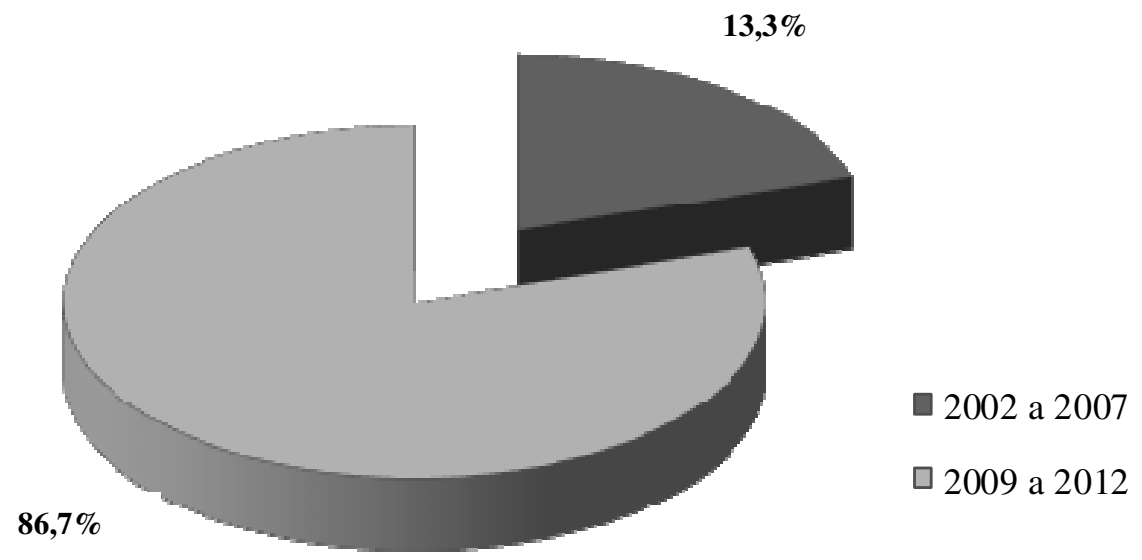

Fonte: Dados da pesquisa bibliográfica. Elaborado pelo autor do artigo em 2014. Teresina/PI. 
Em relação ao local de realização do estudo, podemos observar, na Tabela 1, que houve um destaque para o estado do Piauí, com $27 \%$ da produção e igual percentual para outros estudos internacionais. É importante destacar que a região nordeste foi responsável pelo maior número de trabalhos sobre vacinação, consequentemente centralizando o maior número de publicação sobre o tema nessa região.

\section{Tabela 1 - Estudos revisados sobre a prática da enfermagem na sala de vacina, segundo os estados onde foram realizados. Teresina/PI. $(n=15)$}

\begin{tabular}{lcc}
\hline \multicolumn{1}{c}{ Estados } & $\mathbf{n}$ & $\%$ \\
\hline Piauí & 4 & 27,0 \\
Ceará & 2 & 13,3 \\
São Paulo & 2 & 13,3 \\
Paraná & 1 & 6,1 \\
Pernambuco & 2 & 13,3 \\
Internacionais & 4 & 27,0 \\
\hline Total & 15 & 100 \\
\hline
\end{tabular}

Fonte: Dados da pesquisa bibliográfica. Elaborado pelo autor do artigo em 2014. Teresina/PI.

Dentre os estudos, seis artigos foram publicados em revista de enfermagem, um na Revista Eletrônica de Extensão da URI, dois na revista Brasileira de Epidemiologia, um na Ciências e Saúde Coletiva e os demais em revistas de circulação internacional. No tocante aos resultados encontrados nos estudos, frequentemente um mesmo estudo apresentou diferentes entraves, visto que a maioria dos autores analisaram o contexto geral da sala de vacina, conforme mostra o Quadro 1.

\section{Quadro 1 - Síntese dos estudos que integram a revisão da literatura}

\begin{tabular}{|c|c|c|c|c|}
\hline ANO & REVISTA & AUTOR(ES) & TÍTULO & ENTRAVES ENCONTRADOS \\
\hline 2007 & $\begin{array}{l}\text { Brasileira de } \\
\text { Enfermagem }\end{array}$ & $\begin{array}{l}\text { ARAÚJO, T. M. } \\
\text { E. et al. }\end{array}$ & $\begin{array}{l}\text { Vacina contra influenza: } \\
\text { conhecimentos, atitudes e } \\
\text { práticas de idosos em Teresina. }\end{array}$ & $\begin{array}{l}\text { Promover a participação dos } \\
\text { profissionais de enfermagem no } \\
\text { planejamento, execução } \\
\begin{array}{l}\text { avaliação das ações de } \\
\text { vacinação. }\end{array}\end{array}$ \\
\hline 2007 & $\begin{array}{l}\text { Brasileira de } \\
\text { Enfermagem }\end{array}$ & $\begin{array}{l}\text { ARAÚJO, T. M. } \\
\text { E.; } \\
\text { CARVALHO, P. } \\
\text { M. G.; VIEIRA, } \\
\text { R. D. F. }\end{array}$ & $\begin{array}{l}\text { Análise dos } \\
\text { pós-vacinais } \\
\text { Teresina. }\end{array}$ & $\begin{array}{l}\text { Condutas inadequadas adotadas } \\
\text { pelos profissionais frente aos } \\
\text { Eventos Adversos, Necessidade } \\
\text { de capacitação específica nessa } \\
\text { área para padronização das } \\
\text { rotinas do PNI. }\end{array}$ \\
\hline 2010 & $\begin{array}{l}\text { Acta Paulista } \\
\text { de } \\
\text { Enfermagem }\end{array}$ & $\begin{array}{l}\text { CARVALHO, A. } \\
\text { M. C.; } \\
\text { ARAUJO, T. M. } \\
\text { E. }\end{array}$ & $\begin{array}{l}\text { Fatores associados à cobertura } \\
\text { vacinal em adolescentes }\end{array}$ & $\begin{array}{l}\text { Necessidade de reconstrução de } \\
\text { saberes e práticas com novas } \\
\text { dimensões para a produção de } \\
\text { cuidados em saúde pública, no } \\
\text { tocante a cobertura vacinal. }\end{array}$ \\
\hline
\end{tabular}




\begin{tabular}{|c|c|c|c|c|}
\hline 2011 & $\begin{array}{ll}\text { Eletrônica } & \text { de } \\
\text { Extensão } & \text { da } \\
\text { URI } & \end{array}$ & $\begin{array}{l}\text { MENDES, A. C. } \\
\text { et al. }\end{array}$ & $\begin{array}{l}\text { Vivência de acadêmicos de } \\
\text { enfermagem de uma instituição } \\
\text { de ensino superior de Teresina - } \\
\text { PI na prática em sala de vacina. }\end{array}$ & $\begin{array}{l}\text { Distanciamento do enfermeiro, } \\
\text { como responsável técnico pelo } \\
\text { setor. }\end{array}$ \\
\hline 2009 & Rene Fortaleza & $\begin{array}{l}\text { QUEIROZ, S. } \\
\text { A. et al. }\end{array}$ & $\begin{array}{l}\text { Atuação da equipe } \\
\text { enfermagem na sala } \\
\text { vacinação e suas condições de } \\
\text { funcionamento. }\end{array}$ & $\begin{array}{l}\text { Falta de supervisão na sala de } \\
\text { vacina; } \\
\text { inadequado relacionado à } \\
\text { limpeza do refrigerador da sala } \\
\text { de vacina. }\end{array}$ \\
\hline 2010 & $\begin{array}{l}\text { Cogitare } \\
\text { Enfermagem }\end{array}$ & $\begin{array}{l}\text { FEITOSA, L. } \\
\text { R.; FEITOSA, J. } \\
\text { A.; } \\
\text { CORIOLANO, } \\
\text { M. W. L. }\end{array}$ & $\begin{array}{l}\text { Conhecimentos e práticas do } \\
\text { auxiliar de enfermagem em sala } \\
\text { de imunização. }\end{array}$ & $\begin{array}{l}\text { Falta de treinamento, capacitação } \\
\text { e reflexão, prejudicando a } \\
\text { qualidade do serviço prestado na } \\
\text { sala de vacina. Paralelamente ao } \\
\text { baixo conhecimento, destaca-se } \\
\text { importante lacuna na orientação à } \\
\text { clientela. } \\
\text { Recomenda-se um maior } \\
\text { investimento na educação } \\
\text { permanente desses profissionais. }\end{array}$ \\
\hline 2011 & $\begin{array}{l}\text { Ciências e } \\
\text { saúde coletiva }\end{array}$ & $\begin{array}{l}\text { LUNA, G. M. et } \\
\text { al. }\end{array}$ & $\begin{array}{l}\text { Aspectos relacionados à } \\
\text { administração e conservação de } \\
\text { vacinas em centros de saúde no } \\
\text { Nordeste do Brasil. }\end{array}$ & $\begin{array}{l}\text { Evidência de sucessão de falhas } \\
\text { que podem comprometer a } \\
\text { credibilidade dos imunobiológicos } \\
\text { Necessidade de educação } \\
\text { permanente dos profissionais. }\end{array}$ \\
\hline 2009 & $\begin{array}{l}\text { Atenção } \\
\text { Primária } \\
\text { Saúde }\end{array}$ & $\begin{array}{l}\text { ARAÚJO, A. C. } \\
\text { M.; SILVA, M. } \\
\text { R. F.; FRIAS, P. } \\
\text { G. }\end{array}$ & $\begin{array}{l}\text { Avaliação da Rede de Frio do } \\
\text { Programa Municipal de } \\
\text { Imunização do Distrito Sanitário } \\
\text { IV do Município do Recife. }\end{array}$ & $\begin{array}{l}\text { Ausência de supervisões, } \\
\text { monitoramento e avaliação nas } \\
\text { salas de vacina. }\end{array}$ \\
\hline 2006 & $\begin{array}{l}\text { Brasileira de } \\
\text { Epidemiologia }\end{array}$ & $\begin{array}{l}\text { ARANDA, C. M. } \\
\text { S. S.; } \\
\text { MORAES, J. C. }\end{array}$ & $\begin{array}{l}\text { Rede de frio para a conservação } \\
\text { de vacinas em unidades públicas } \\
\text { do município de São Paulo: } \\
\text { conhecimento e prática. }\end{array}$ & $\begin{array}{l}\text { Falta de conhecimento da equipe } \\
\text { sobre inativação dos } \\
\text { imunobiológicos } \\
\text { congelamento. }\end{array}$ \\
\hline 2010 & $\begin{array}{l}\text { Epidemiologia } \\
\text { em Serviços de } \\
\text { Saúde }\end{array}$ & $\begin{array}{l}\text { MELO, G. } \mathrm{K} . \\
\text { M.; OLIVEIRA } \\
\text { J. } \\
\text { ANDRADE, M.; } \\
\text { S. }\end{array}$ & $\begin{array}{l}\text { Aspectos relacionados à } \\
\text { conservação de vacinas nas } \\
\text { unidades básicas de saúde da } \\
\text { cidade do Recife - Pernambuco. }\end{array}$ & $\begin{array}{l}\text { Falta de capacitação da equipe } \\
\text { de sala de vacina. } \\
\text { Conhecimento equivocado em } \\
\text { relação à conservação de } \\
\text { vacinas. }\end{array}$ \\
\hline 2009 & $\begin{array}{l}\text { Acta Paulista } \\
\text { de } \\
\text { Enfermagem }\end{array}$ & $\begin{array}{l}\text { OLIVEIRA, } \mathrm{V} . \\
\text { C. et al. }\end{array}$ & $\begin{array}{l}\text { Prática da enfermagem na } \\
\text { conservação de vacinas. }\end{array}$ & Falta de capacitação da equipe. \\
\hline 2009 & $\begin{array}{l}\text { Gaceta } \\
\text { Sanitaria }\end{array}$ & $\begin{array}{l}\text { BARBER- } \\
\text { HUESO, C. et } \\
\text { al. }\end{array}$ & $\begin{array}{l}\text { La cadena de frío vacunal en un } \\
\text { departamento de salud de la } \\
\text { Comunidad Valenciana. }\end{array}$ & $\begin{array}{l}\text { Conhecimento equivocado sobre } \\
\text { conservação de vacinas. }\end{array}$ \\
\hline 2002 & $\begin{array}{l}\text { Española de } \\
\text { Salud Publica }\end{array}$ & $\begin{array}{l}\text { MOLINA, P. O. } \\
\text { et al. }\end{array}$ & $\begin{array}{l}\text { Cadena del frío para la } \\
\text { conservación de las vacunas en } \\
\text { los centros de atención primaria } \\
\text { de un área de Madrid: } \\
\text { mantenimiento y nível de } \\
\text { conocimientos. }\end{array}$ & $\begin{array}{l}\text { Conhecimento equivocado sobre } \\
\text { conservação de vacinas. }\end{array}$ \\
\hline 2011 & $\begin{array}{l}\text { Nagoya } \\
\text { Journal } \\
\text { Medical } \\
\text { Science }\end{array}$ & $\begin{array}{l}\text { WIDSANUGOR } \\
\text { N, O. et al. }\end{array}$ & $\begin{array}{l}\text { Healthcare workers' knowledge } \\
\text { and practices regarding } \\
\text { expanded program on } \\
\text { immunization in Kalasin. }\end{array}$ & $\begin{array}{l}\text { Conhecimento equivocado sobre } \\
\text { acondicionamento de vacinas. }\end{array}$ \\
\hline 2011 & $\begin{array}{l}\text { American } \\
\text { Journal of } \\
\text { Public Health }\end{array}$ & $\begin{array}{l}\text { MCCOLLOSTE } \\
\text { R, P.; } \\
\text { VALLBONA, C. }\end{array}$ & $\begin{array}{l}\text { Graphic-output temperature data } \\
\text { loggers for monitoring vaccine } \\
\text { refrigeration: implications for } \\
\text { pertussis. }\end{array}$ & $\begin{array}{l}\text { Conhecimento equivocado sobre } \\
\text { conservação de vacinas. }\end{array}$ \\
\hline
\end{tabular}

Fonte: Dados da pesquisa bibliográfica. Elaborado pelo autor do artigo em 2014. Teresina/PI.

Nos 15 artigos analisados, observam-se resultados semelhantes que apontam para a necessidade de educação continuada dos profissionais de sala de vacina, pois foram identificados, em todas as publicações, conhecimentos insuficientes no setor supracitado. 


\section{DISCUSSÃO}

Quando se inseriu na estratégia de busca a qualidade das ações realizadas nas salas de vacina no Brasil, chegou-se a um resultado de 15 artigos, o que significa dizer que existe um número escasso de publicações específicas relacionadas ao conhecimento dos profissionais em sala de vacina. A enfermagem publicou quase mil artigos científicos nos últimos seis anos, no entanto apenas 15 na temática que explora esse tipo de conhecimento.

Percebe-se que a produção científica levantada no presente estudo, concentrou-se nos anos de 2009 a 2012, o que demonstra um aumento do interesse na verificação do conhecimento dos profissionais de sala de vacina e, consequentemente, a qualidade de suas ações, nos últimos cinco anos; o que pode ser explicado pelo fato do conhecimento em vacinas ser denso, complexo e dinâmico, de modo que necessita de atualizações frequentes, bem como o processo sistemático e contínuo de supervisão por parte do enfermeiro. As vacinas estão em constante mudanças, aperfeiçoamento, descoberta e recombinação, exigindo do profissional a busca por informações, aperfeiçoamento e prática acerca da temática.

Entre os estados que mais publicaram artigo sobre sala de vacina estão o Piauí (ARAÚJO et al., 2007; ARAÚJO; CARVALHO; VIEIRA, 2007; CARVALHO; ARAÚJO, 2010; MENDES et al., 2011), com destaque para a região Nordeste (QUEIROZ et al., 2009; LUNA et al., 2011; ARAÚJO; SILVA; FRIAS, 2009; MELO; OLIVEIRA; ANDRADE, 2010), totalizando número de publicações expressivo no universo pesquisado, o que demonstra que a região nordeste é a que mais pesquisa conhecimento sobre rotina de sala de vacina.

A metodologia quantitativa foi significativamente mais usada nos artigos selecionados.

Os objetivos dos estudos foram os mais diversificados, porém somente uma das publicações fazia uma análise completa da qualidade do trabalho da sala de vacina, levando em consideração temperatura de acondicionamento dos imunobiológicos, condições dos equipamentos, estrutura da sala, controle da temperatura com preenchimento do mapa, preparo e administração da vacina, posicionamento da criança para administração, tempo de utilização dos imunobiológicos após abertura dos frascos, utilização correta de agulhas, destino do lixo gerado na sala de vacina, higiene das mãos, educação continuada, qualificação da equipe, presença do enfermeiro na sala de vacina, preenchimento correto dos impressos, conduta frente aos efeitos adversos (MENDES et al., 2011).

Sobre o conhecimento dos profissionais, inúmeros artigos demonstraram necessidade urgente de atualização, visto que os profissionais envolvidos com a prática de vacinação e, de modo especial, aqueles que atuam em sala de vacina, precisam ter a máxima segurança na realização de todos os procedimentos referentes a essa prática, o que pode ser possível por meio dos processos de qualificação, os quais precisam adequar-se à nova realidade do PNI.

Considerando a atuação desses profissionais de enfermagem em sala de vacina, 
destacam-se repetidamente conhecimento desatualizado e inadequado em relação à correta temperatura de acondicionamento dos imunobiológicos que não podem ser expostos a baixas temperaturas (ARANDA; MORAES, 2006; OLIVEIRA et al., 2009; FEITOSA; FEITOSA; CORIOLANO, 2010), bem como o inadequado conhecimento para ambientação das bobinas recicláveis e acondicionamento dos imunobiológicos no refrigerador (MELO; OLIVEIRA; ANDRADE, 2010).

Cabe mencionar que o conhecimento sobre as vacinas é muito dinâmico, exigindo atualização constante, item não observado nesta investigação (MELO; OLIVEIRA; ANDRADE, 2010; OLIVEIRA et al., 2009), demonstrando conhecimento sobre vacinas muito aquém do preconizado pelo PNI. Ressalta-se, ainda, que conhecimento insuficiente para a prática de vacina não é realidade exclusiva do Brasil. Estudos internacionais apontam conhecimento inadequado dos profissionais relacionados à temperatura correta de acondicionamento das vacinas, sob pena de alteração de seu poder imunogênico (BARBER-HUESO et al., 2009; MOLINA et al., 2002; WIDSANUGORN et al., 2011; MCCOLLOSTER; VALLBONA, 2011).

No entanto, observa-se que algumas publicações enfatizam mais a conservação dos imunobiológicos, enquanto que outras mais as condutas frente aos eventos adversos. Isso se torna preocupante, visto que é uma abordagem limitada em relação a um tema de extrema importância e que se faz necessário conhecimento amplo e detalhado referente ao cumprimento, com rigor, de todas as ações que englobam o PNI. Uma vez que o não atendimento aos requisitos do manual de imunização pode comprometer seriamente a vida dos usuários, bem como a qualidade e credibilidade do imunobiológico.

Cabe destacar que em todas as publicações exploradas foi possível observar, de alguma forma falhas no cumprimento das ações estabelecidas pelos manuais de vacinação, destacando-se como unanimidade a necessidade de qualificação dos profissionais, tendo em vista que ocorrem constantes mudanças nos calendários vacinais e os treinamentos para os profissionais não acontecem na mesma proporção e velocidade.

\section{CONCLUSÃO}

O controle das doenças imunopreviníveis por meio dos programas de imunização constitui uma das medidas mais eficazes para a promoção da saúde das pessoas, fazendose necessário um preparo obrigatório dos profissionais das salas de vacinas, o que vem deixando muito a desejar conforme publicações analisadas neste estudo.

A produção científica sobre conhecimento dos profissionais da sala de vacina é pouco significativa e mais escassa ainda quando se trata de uma abordagem levando-se em consideração o conhecimento referente a todos os aspectos envolvidos, que garantem cumprimento do objetivo do PNI. Destaca-se a constatação como aspecto negativo, visto que uma assistência de qualidade deve ser essencialmente pautada na qualificação contínua dos profissionais, fato não observado na análise realizada. 
Mediante esta revisão bibliográfica, percebe-se que não há a valorização necessária em relação a essas questões por parte de enfermeiro, ou, pelo menos, existem poucas produções científicas sobre o tema, fato também observado na análise realizada.

Foi possível identificar, no espaço de tempo estabelecido entre 2002 e 2012, que não existe um número significativo de produções específicas da enfermagem em sala de vacina. O que não significa que não estejam sendo produzidos conhecimentos nessa área, porém existe a necessidade de enfatizar a grandeza dessa temática na saúde do nosso país, já que a vacinação segura promove o controle das doenças imunizáveis. Assim, produzir mais nesta temática reflete também valorizar o cuidado e a orientação de enfermagem, uma vez que é o enfermeiro quem coordena essas ações de imunização.

\section{REFERÊNCIAS}

ARANDA, C. M. S. S.; MORAES, J. C. Rede de frio para a conservação de vacinas em unidades públicas do município de São Paulo: conhecimento e prática. Rev. bras. epidemiol, São Paulo, v. 9, n. 2, p. 172-85, 2006.

ARAÚJO, T. M. E. et al. Vacina contra influenza: conhecimentos, atitudes e práticas de idosos em Teresina. Rev. bras. enferm, São Paulo, v. 60, n. 1, p. 223-228, jan. 2007.

ARAÚJO, T. M. E.; CARVALHO, P. M. G.; VIEIRA. R. D. F. Análise dos eventos adversos pós-vacinais ocorridos em Teresina. Rev. bras. Enferm, São Paulo, v. 60, n. 4, p. 444-448, fev. 2007.

ARAÚJO, A. C. M.; SILVA, M. R. F.; FRIAS, P. G. Avaliação da Rede de Frio do Programa Municipal de Imunização do Distrito Sanitário IV do Município do Recife. Rev. APS, Juiz de Fora, v. 12, n. 3, p. 167-176, set. 2009.

BARBER-HUESO, C. et al. La cadena de frío vacunal en un departamento de salud de la Comunidad Valenciana. Gac. sanit., Barcelona, v. 23, n. 2, p. 139-143. 2009.

BRASIL. Ministério da Saúde. Capacitação de pessoal em sala de vacina. Manual do Monitor. Brasília: Ministério da Saúde, 2011.

Ministério da Saúde. Manual de rede de frio. Brasília: Ministério da Saúde, 2013.

CARVALHO, A. M. C.; ARAÚJO, T. M. E. Fatores associados à cobertura vacinal em adolescentes. Acta paul. enferm, São Paulo, v. 23, n. 6, p. 103-123, abr. 2010.

FEITOSA, L. R.; FEITOSA, J. A.; CORIOLANO, M. W. L. Conhecimentos e práticas do auxiliar de enfermagem em sala de imunização. Rev. Cogitare Enferm, Pernambuco, v. 15, n. 4, p. 123-12, jun. 2010.

LUNA, G. M. et al. Aspectos relacionados à administração e conservação de vacinas em centros de saúde no Nordeste do Brasil. Ciênc. saúde coletiva, Rio de Janeiro, v. 16, n. 2, p. 513-521, fev. 2011. 
MCCOLLOSTER, P.; VALLBONA, C. Graphic-output temperature data loggers for monitoring vaccine refrigeration: implications for pertussis. Am. j. public health, Washington, v. 101, n. 1, p. 46-48, jan. 2011.

MELO, G. K. M.; OLIVEIRA J. V.; ANDRADE, M. S. Aspectos relacionados à conservação de vacinas nas unidades básicas de saúde da cidade do Recife - Pernambuco. Epidemiol. Serv. Saúde, Brasília, v. 19, n. 1, p. 26-33, mar. 2010.

MENDES, A. C. et al. Vivência de acadêmicos de enfermagem de uma instituição de ensino superior de Teresina - PI na prática em sala de vacina. Vivências, Santo Ângelo, v. 13, n. 7 , p. 209-217, out. 2011.

MOLINA, P. O. et al. Cadena del frío para la conservación de las vacunas en los centros de atención primaria de un área de Madrid: mantenimiento y nível de conocimientos. Rev. esp. salud pública, Madrid, v. 76, n. 4, p. 131-3, 2002.

OLIVEIRA, V. C. et al. Prática da enfermagem na conservação de vacinas. Acta paul. Enferm, São Paulo, v. 22, n. 6, p. 210-205, dez. 2009.

QUEIROZ, S. A. et al. Atuação da equipe de enfermagem na sala de vacinação e suas condições de funcionamento. Rev. Rene, Fortaleza, v. 10, n. 4, p. 210-200, out. 2009.

SOUZA, M. T.; SILVA, M. D.; CARVALHO, R. Revisão integrativa: o que é e como fazer. Einstein, São Paulo, v. 8, n. 1, p. 102-106, fev. 2010.

WIDSANUGORN, O. et al. Healthcare workers' knowledge and practices regarding expanded program on immunization in Kalasin, Thailand. Nagoya j. med. sci., Nagoya, v. 73, n. 4, p. 177-185. 2011. 